\title{
Dysfunctional HCN ion channels in neurological diseases
}

\author{
Jacopo C. DiFrancesco ${ }^{1,2 *}$ and Dario DiFrancesco ${ }^{3}$ \\ ${ }^{1}$ Department of Neurophysiology, Foundation Neurological Institute C. Besta, Milano, Italy, ${ }^{2}$ Department of Neurology, San \\ Gerardo Hospital and Laboratory of Neurobiology, Milan Center for Neuroscience, University of Milano-Bicocca, Monza, \\ Italy, ${ }^{3}$ The PaceLab, Department of Biosciences, University of Milano, Milano, Italy
}

\section{OPEN ACCESS}

Edited by:

Maria Cristina D'Adamo, University of Perugia, Italy

Reviewed by: Qian Sun, Columbia University, USA Eric Accili, The University of British Columbia, Canada

*Correspondence: Jacopo C. DiFrancesco, Department of Neurology, San Gerardo Hospital and Laboratory of Neurobiology, Milan Center for Neuroscience, Department of Surgery \& Translational Medicine,

University of Milano-Bicocca, Via Cadore, 48 - 20900 Monza, Italy jacopo.difrancesco@unimib.it; jacopo.difrancesco@gmail.com

Received: 19 December 2014 Paper pending published: 21 January 2015 Accepted: 18 February 2015 Published: 10 March 2015

Citation:

DiFrancesco JC and DiFrancesco D (2015) Dysfunctional HCN ion channels in neurological diseases. Front. Cell. Neurosci. 9:71. doi: 10.3389/fncel.2015.00071
Hyperpolarization-activated cyclic nucleotide-gated $(\mathrm{HCN})$ channels are expressed as four different isoforms (HCN1-4) in the heart and in the central and peripheral nervous systems. HCN channels are activated by membrane hyperpolarization at voltages close to resting membrane potentials and carry the hyperpolarization-activated current, dubbed $I_{f}$ (funny current) in heart and $I_{h}$ in neurons. HCN channels contribute in several ways to neuronal activity and are responsible for many important cellular functions, including cellular excitability, generation, and modulation of rhythmic activity, dendritic integration, transmission of synaptic potentials, and plasticity phenomena. Because of their role, defective HCN channels are natural candidates in the search for potential causes of neurological disorders in humans. Several data, including growing evidence that some forms of epilepsy are associated with HCN mutations, support the notion of an involvement of dysfunctional HCN channels in different experimental models of the disease. Additionally, some anti-epileptic drugs are known to modify the activity of the $I_{h}$ current. HCN channels are widely expressed in the peripheral nervous system and recent evidence has highlighted the importance of the HCN2 isoform in the transmission of pain. HCN channels are also present in the midbrain system, where they finely regulate the activity of dopaminergic neurons, and a potential role of these channels in the pathogenesis of Parkinson's disease has recently emerged. The function of $\mathrm{HCN}$ channels is regulated by specific accessory proteins, which control the correct expression and modulation of the neuronal $\mathrm{l}_{\mathrm{h}}$ current. Alteration of these proteins can severely interfere with the physiological channel function, potentially predisposing to pathological conditions. In this review we address the present knowledge of the association between HCN dysfunctions and neurological diseases, including clinical, genetic, and physiopathological aspects.

Keywords: epilepsy, HCN channels, HCN mutation, $I_{h}$ current, seizure, Parkinson's disease, pain

\section{Introduction}

The class of hyperpolarization-activated cyclic nucleotide-gated (HCN) channels comprises four human isoforms (HCN1-4) and belongs to the superfamily of voltage-dependent potassium (Kv) and cyclic nucleotide-gated (CNG) channels. HCN channels are unique in that they are dually activated by voltage hyperpolarization and intracellular cAMP (DiFrancesco, 1999, 2006; Barbuti et al., 2007a; Baruscotti et al., 2010). 
Since they are constitutively open at voltages near to the resting membrane potential and, being permeable to both $\mathrm{Na}^{+}$and $\mathrm{K}^{+}$ions, carry at these voltages an inward current, HCN channels are well equipped to control voltagedependent mechanisms, such as neuronal excitability, and related functions.

In neurons, HCN channels carry the hyperpolarization activated $\left(\mathrm{I}_{\mathrm{h}}\right)$ current. The four isoforms are differently distributed: HCN1, 2 and 4 are widely expressed and contribute to the generation of neuronal activity, while the role of HCN3 is less clear. In neurons, HCN are responsible for several important cellular functions, including the contribution to cellular excitability, and plasticity phenomena in the brain. One of the most specific functions of HCN channels is their involvement in the generation and modulation of rhythmic activity, which is typified by the role they play in the spontaneous activity and frequency control of pacemaker cells of the heart (DiFrancesco, 1993; Pape, 1996; Robinson and Siegelbaum, 2003; Barbuti et al., 2007a).

As expected from the established role of HCN channels in neurons, in the last few years new evidence has emerged linking HCN channels' dysfunctions to different neurological disorders, in accordance with the view that altered pacemaker activity can act as a strong pathogenic mechanism.

The vast majority of such data have been obtained in the field of epilepsy (Dyhrfjeld-Johnsen et al., 2009; Baruscotti et al., 2010; DiFrancesco et al., 2011; Reid et al., 2012; Shah et al., 2013). Together with data from animal models showing that $\mathrm{HCN}$ dysfunction can lead to epileptogenesis, evidence is accumulating to indicate that some inheritable forms of epilepsy can be associated in human patients with HCN genetic mutations leading to altered $\mathrm{I}_{\mathrm{h}}$ current function and increased neuronal excitability. Although a comprehensive picture of the genetic basis of epilepsy is far from complete, these new data contribute importantly to a deeper understanding of the processes responsible for causative changes in neuronal excitability.

As well as in the central nervous system (CNS), HCN channels are also widely expressed in the peripheral nervous system (PNS), where their function is important in the perception, modulation, and transmission of sensory signals, including pain. Recent data demonstrate a central role of the HCN2 isoform in the transmission of neuropathic and inflammatory pain (Emery et al., 2011).

Moreover, HCN channels are expressed in the midbrain system, where they control the oscillatory activity of dopaminergic neurons. In line with this evidence, a new potential role of $\mathrm{HCN}$ channels dysfunction is also emerging in the pathogenesis of Parkinson's disease (PD).

Full knowledge of the pathogenic mechanisms underlying these debilitating neurological diseases and the role played by aberrant $\mathrm{HCN}$ channel function is not at hand. However, a more comprehensive understanding is desirable in many respects, particularly in view of the possibility to identify new potential therapeutic strategies (DiFrancesco and Borer, 2007; Dunlop et al., 2009; Wickenden et al., 2009; Postea and Biel, 2011; Reid et al., 2012; Shah et al., 2013).
In this review we address the current knowledge of $\mathrm{HCN}$ channels properties and their role of dysfunctional behavior in human neurological diseases.

\section{Epilepsy}

Epilepsy is a neurological disorder characterized by recurrent paroxysmal episodes of brain electrical dysfunction. Seizures are caused by an excessive and sudden electrical discharge of neurons of the CNS. Epilepsy is a common disorder, affecting about $4 \%$ of the general population, often involving children, and young adults, with a very high cost to society in terms of direct expenses for the public health service and disabilities. Epilepsy affects at least 50 million people worldwide, with a global mortality rate higher than cancer.

The cause of the disease remains unknown in about $60 \%$ of cases, which are referred to as "idiopathic." About $30 \%$ of idiopathic epilepsies are inheritable and affect several members of the family. Current research reveals that several types of epilepsy, both familiar, and sporadic, have a genetic component mainly linked to mutations in genes encoding either voltage-gated $\left(\mathrm{Na}^{+}\right.$, $\mathrm{Ca}^{2+}$, and $\mathrm{K}^{+}$) or ligand-gated (GABAA and cholinergic nicotinic receptor) channels (Avanzini et al., 2007; Mantegazza et al., 2010; Thomas and Berkovic, 2014).

The recognition that certain epileptic syndromes are channelopathies has opened new perspectives in the understanding of the molecular pathophysiology of seizure disorders. Following findings of causative links between epileptic syndromes and mutations in voltage-gated or ligand-gated channels, the family of ion channels involved in epileptogenic channelopathies has grown rapidly. Together with the ion channel types whose involvement has been established early, another family that according to more recent data play a role in the pathogenesis of inheritable epilepsy is the family of HCN channels. Because of their established role in modulating neuronal excitability, a modification of the function of HCN channels is clearly potentially able to cause uncontrolled action potential firing and provide a background setting for the development of epilepsy (Baruscotti et al., 2010).

In the last few years, evidence for a potential relevant role of HCN channels in the pathogenesis of epilepsy has grown considerably. Many results derive from animal models, but a substantial bulk of recent data shows that $H C N$ dysfunctional genetic mutations associated with epilepsy are also expressed in human patients.

\section{Animal Models}

Several data supporting a link between functional alteration of HCN channels and epileptogenesis have been obtained by studies of HCN1 and HCN2 knock-out mouse models.

For example, cortical excitability and epileptogenesis are enhanced by loss of HCN1 expression and abolishment of $\mathrm{I}_{\mathrm{h}}$ in HCN1-null mice, since lack of this channel increases the dendritic input resistance in cortical neurons, leading to greater synaptic integration, and firing (Huang et al., 2009). In the same HCN1null mouse model, greater dendritic excitability, and temporal 
summation were also observed in hippocampal CA1 pyramidal neurons (Nolan et al., 2004; Tsay et al., 2007). In another study, in agreement with a role for $\mathrm{I}_{\mathrm{h}}$ downregulation in epileptogenesis, HCN2-deficient mice generated by a global knockout model were shown to exhibit spontaneous absence seizures (Ludwig et al., 2003). Generalized spike-wave absence seizures were also observed in spontaneous HCN2 mutant (apathetic) mice, where channel proteins are truncated at the $\mathrm{C}$ terminus and poorly expressed (Chung et al., 2009). Moreover, different genetic models of absence epilepsy such as WAG/Rij and GABAA 2 2(R43Q) revealed an altered expression of $\mathrm{HCN} 1$ associated with the occurrence of seizures (Kole et al., 2007; Phillips et al., 2014).

The remodeling of HCN channels expression also provides an important contribution to the epileptogenic network and seizure production. In the immature animal brain, sustained febrile seizures (FS) increase neuronal excitability and the predisposition to develop epilepsy in adults, by modifying the membrane expression of HCN isoforms 1 and 2 (Dube et al., 2000; Brewster et al., 2002). Seizures provoke a prolonged remodeling of $\mathrm{HCN} 1$ and $\mathrm{HCN} 2$ expression, supporting the presence of a transcriptional channelopathy involving HCN channels which contributes to the development of epilepsy (Richichi et al., 2008). Similar observations can be made in the epileptic human brain tissue, where the expression of $\mathrm{HCN}$ channels is altered, indicating that the $\mathrm{HCN}$ isoforms are dynamically regulated in humans in vivo, as well as in the experimental hippocampal epilepsy (Bender et al., 2003).

In accordance with the observations above, different models of temporal lobe epilepsy (TLE), the most frequent form of partial epilepsy in humans, revealed a significant downregulation of HCN channels expression following pharmacological status epilepticus (SE), with increased neuronal excitability. This could have important implications for both the process of epileptogenesis and maintenance of the epileptic state (Jung et al., 2007, 2011; Powell et al., 2008).

\section{Human Patients}

Despite the availability of a substantial amount of experimental data on animal models supporting the role played by HCN channels in the pathogenesis of epilepsy, evidence from human patients is still limited.

Tang et al. (2008), published a pilot study of 84 patients with a non-inheritable form of idiopathic generalized epilepsy (IGE) and searched for mutations in $\mathrm{HCN} 1$ and $\mathrm{HCN} 2$ in this cohort. They identified, among others, a single point mutation in the C-linker region of HCN2, affecting a highly conserved and functionally relevant residue (R527Q). However, a conclusive proof that this mutation is causative was lacking, since functional studies did not show statistically significant differences between the properties of mutant and wild-type channels. Dibbens and colleagues later reported that in a population of children with FS and genetic epilepsy with FS plus (GEFS+), the rate of appearance of an $\mathrm{HCN} 2$ variant characterized by a triple proline deletion (delPPP, p.719-721) was significantly higher than in the general population. Heterologous expression studies indicated that delPPP channels carry a $35 \%$ larger $\mathrm{I}_{\mathrm{h}}$ current than wild-type channels, suggesting that delPPP behaves as a gain-of-function mutation (Dibbens et al., 2010). However, while statistical significance is a necessary condition to demonstrate a link between mutation and diseased phenotype, the study did not address how the mutation alters the functional properties of channels and how they impact neuronal excitability.

In a more recent study based on $H C N$ candidate-gene screening of a small cohort of patients $(n=113)$, our group characterized for the first time a loss-of-function $H C N 2$ mutation that could be shown from functional studies to be directly involved in human epilepsy (Figure 1). This study led to the identification, in a generalized epilepsy patient, of a homozygous recessive mutation located in the C-linker region of HCN2 (E515K; Figure 1A) that determines an almost complete loss of activity of the mutated channel, leading to a significant increase in the activity of neuronal discharge, and of neuronal excitability (DiFrancesco et al., 2011). As shown in Figure 1B, homozygous expression of E515K mutated channels in acutely isolated newborn rat cortical neurons led to a large negative shift of the $\mathrm{I}_{\mathrm{h}}$ current activation curve relative to wild-type channels, while heterozygous expression of wild type/E515K channels did not (Figure 1B), indicating that only recessive inheritance of the mutation will display loss-of-function properties. In the neuronal model, following administration of increasing doses of depolarizing current (range 10-50 pA), the transfection of both wild type and heterozygous mutant channel reduced frequency discharge, compared to control conditions (empty-vector transfection) and transfection of homozygous mutant channels (Figure 1C). These functional data confirm that HCN2 expression stabilizes excitability, and that removal of HCN2 contribution in homozygous E515K variants is associated with increased excitability, a condition predisposing to epilepsy.

Later work from Nakamura and colleagues reported the finding in two unrelated patients with FS of a mutation in HCN2 (S126L) which endowed channels with a higher than normal temperature sensitivity. Functional investigation showed that the mutation leads to an increased $\mathrm{I}_{\mathrm{h}}$ availability at high temperatures $\left(38^{\circ} \mathrm{C}\right)$, which may contribute to hyperthermia-induced neuronal hyperexcitability in FS (Nakamura et al., 2013).

More recently, using an exome sequencing approach, de novo mutations in HCN1 have been identified for the first time in early infantile epileptic encephalopathy (EIEE; Nava et al., 2014a). EIEE is a severe disease affecting children within the first year of life which resembles the spectrum of Dravet syndrome and had been previously associated with mutations in SCN1A sodium channel and PCDH19 (Depienne et al., 2009a,b). According to the authors, the mutated $\mathrm{HCN} 1$ residues associated with EIEE are all localized in the intracellular part of the channel, although one of them (S272) lies apparently well within the S4 domain according to previously reported data (Santoro et al., 1998). Interestingly, HCN1 exon deletions have been previously reported in patients with autism spectrum disorders, but without epilepsy (Nava et al., 2014b). The electrophysiological characterization of the mutations revealed for some of them a gain-offunction effect that could play a dominant-negative effect, interfering with the function of the remaining HCN1 alleles. Taken together, these data suggest that point mutations altering $\mathrm{HCN} 1$ 
A
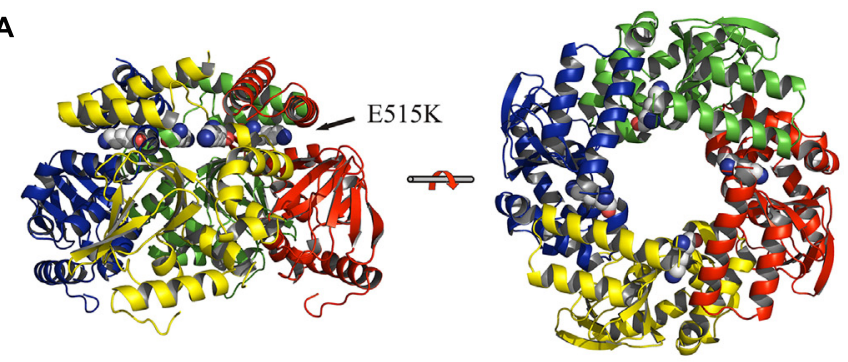

B
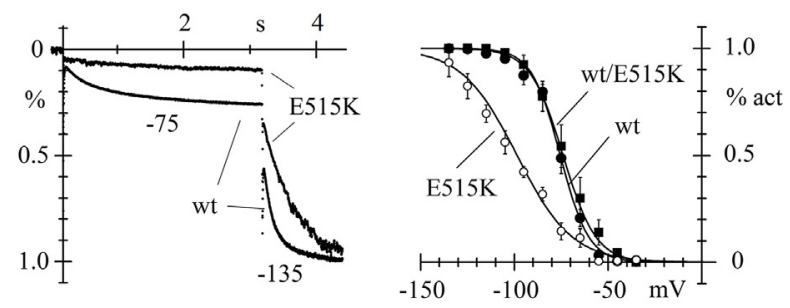

C
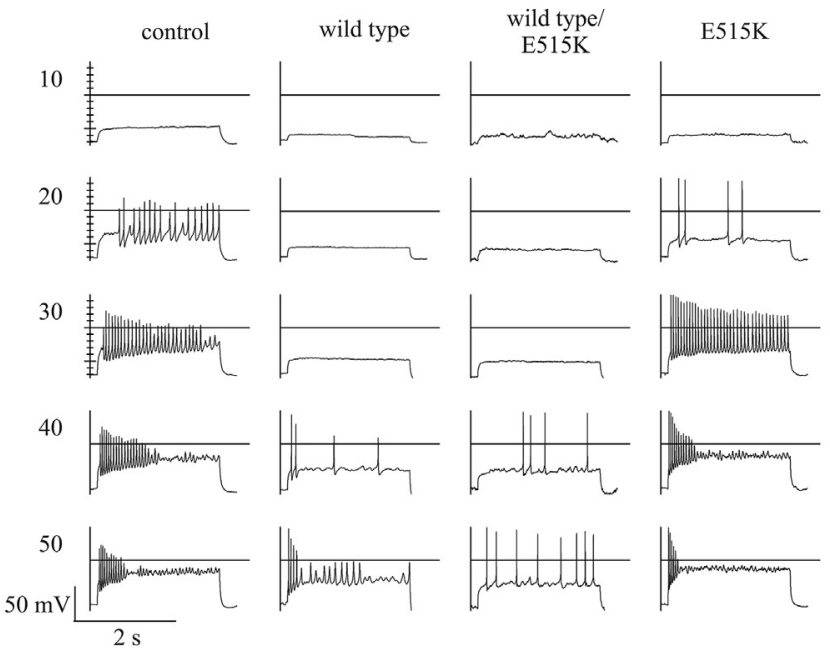

D

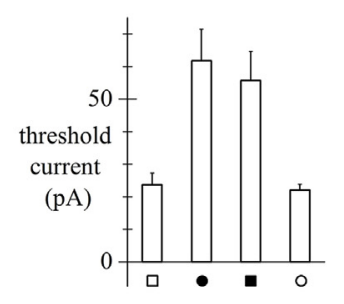

FIGURE 1 | Human HCN2 E515K mutation and its effect on neuronal excitability. 3D structure of hHCN2 C-linker and cyclic nucleotide binding domain (CNBD) tetrameric domains based on X-ray crystallographic data (Zagotta et al., 2003) and plotted as ribbon representation. Views shown are perpendicular (left) and parallel (right) to fourfold axis; E515 residues are drawn as space filling plots; the E515K mutation is located in the C-linker region (A). When overexpressed in neonatal rat cortical neurons, the E515K homozygous mutation leads to a large decrease of $\mathrm{Ih}_{\mathrm{h}}$ in the voltage range of current activation (left), due to a large negative shift of the activation curve (right), when compared to both the wild-type (wt) and the heterozygous mutation channel (wt/E515K) (B). Measurement of the firing rate due to

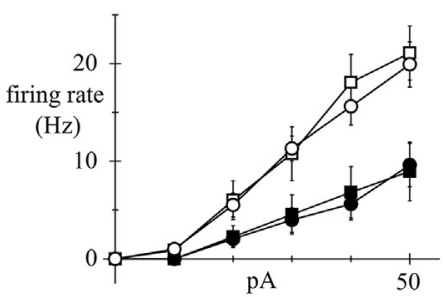

injection of various degrees of depolarizing currents (range 10-50 pA) shows that neonatal rat cortical neurons transfected with either wild type or heterozygous wild-type/mutant channels respond with a much-reduced excitability than neurons transfected with empty vectors (controls) or with homozygous E515K mutant channels (C). Mean threshold current required to trigger action potential firing and mean rate of firing recorded upon current injection in control, wt, wt/E515K, and E515K cells. Neuronal models transfected with homozygous E515K mutant channels, like empty vectors, show a significantly lower threshold current to trigger neuronal action potential and a higher rate of firing action potential, upon depolarizing current injection (D). Data from (DiFrancesco et al., 2011), with permission. 
channel function are poorly tolerated and predispose to neuronal hyperexcitability, but are insufficient by themselves to cause seizure development. This is supported by data from experimental animal models of global HCN1 knockout, characterized by a predisposition to the development of epilepsy, but without spontaneous seizures (Huang et al., 2009; Santoro et al., 2010). These observations suggest that de novo HCN1 mutations are associated with EIEE, a particularly severe neurological phenotype, while there is still no evidence for the involvement of HCN1 in IGE (Tang et al., 2008; Dibbens et al., 2010; DiFrancesco et al., 2011).

Even if exhaustive genetic studies on a large cohort of patients have not yet been performed, according to these preliminary results (Table 1), HCN1 mutations seem to involve infantile, severe, and progressive epileptic encephalopathies, while HCN2 appear to affect patients with normal intellective level, without evidence for a progressive disease. Interestingly, there is no evidence yet for the involvement in epilepsy of dysfunctional mutations in $\mathrm{HCN} 4$, despite this isoform is widely expressed in the human brain. Figure 2 illustrates the rough sequence positions of mutations of HCN1 and HCN2 channels so far proposed to be associated with epilepsy.

\section{Hcn-Accessory Proteins}

Dysfunctional behavior of the $\mathrm{I}_{\mathrm{h}}$ current can also be mediated by changes in the $\mathrm{HCN}$-accessory proteins. This is a set of proteins (such as MiRP1, Filamin A, TRIP8b, Caveolin 3, tamalin, Mint2, S-SCAM, KCR1) that affect the correct function of $\mathrm{HCN}$ ion channels by interaction at different levels (Yu et al., 2001; Gravante et al., 2004; Kimura et al., 2004; Santoro et al., 2004, 2009; Barbuti et al., 2007b; Michels et al., 2008; Biel et al., 2009; Noam et al., 2014). It is known for example that the targeted deletion of MiRP1 leads to the downregulation of HCN channel function, which in turn is associated with increased excitability in neurons (Ying et al., 2012). A more direct confirmation of functional interaction between channels and ancillary proteins comes from a study investigating a single patient with long QT syndrome (LQT6) who carried the M54T mutation in MiRP1 (Nawathe et al., 2013). Since this patient was also symptomatic for bradycardia, a condition not normally found in LQT patients, the study explored possible changes in $\mathrm{HCN}$ function and found that the M54T mutation indeed causes the HCN4 current density to decrease by about $80 \%$, in agreement with the patient's bradycardia.
To our knowledge there is no established evidence so far for a direct link between defective $\mathrm{HCN}$ accessory proteins and neuronal hyperexcitability in epilepsy patients, although the possibility for such a dependence is likely to be significant.

\section{Anti Epileptic Drugs}

An observation emphasizing the clinical relevance of the concept of HCN-dependent epilepsy is that some anti-epileptic drugs (AEDs) currently used for the treatment of patients have been reported to modify the activity of the $\mathrm{I}_{\mathrm{h}}$ current. For example lamotrigine, widely used in clinical practice, reduces action potential firing and excitability in dendrites as a result of an increased $\mathrm{I}_{\mathrm{h}}$, which provides an important potential mechanism for its antiepileptic action (Poolos et al., 2002). In addition, the antiepileptic drug gabapentin increases $\mathrm{I}_{\mathrm{h}}$ in CA1 pyramidal neurons (Surges et al., 2003) and acetazolamide, an inhibitor of carbonic anhydrase that has been used to treat epilepsy, can increase $\mathrm{I}_{\mathrm{h}}$ through intracellular alkalinization (Munsch and Pape, 1999). These data confirm the role played by the $\mathrm{I}_{\mathrm{h}}$ current in the epileptogenic process.

\section{Pain}

The sensation of pain is evoked when the body is exposed to a potentially dangerous stimulus. This determines nerve membrane depolarization and generation of action potentials from peripheral nociceptors to CNS. Pain can generally be divided into inflammatory and neuropathic.

Acute inflammatory pain has the crucial role of protecting the body from external damage, thus lowering the risk of further damage or infection of an injured area. It is triggered by the release of inflammatory factors, such as prostaglandin $\mathrm{E}_{2}$ $\left(\mathrm{PGE}_{2}\right)$ and enhances the ability of nociceptive nerve terminals to generate action potentials, thus causing local hypersensitivity (Linley et al., 2010). Typically, when the stimulus ceases, nociceptive pain perception disappears. However, in certain circumstances, such as diabetes, or herpes zoster infection, inflammatory pain becomes chronic, and seriously impairs the quality of life of patients.

Neuropathic pain is a long-lasting pain state caused by nerve injury, poorly treated by pharmacological drugs. Patients frequently complain of painful spontaneous sensations, evoked by normally harmless stimuli. While a major role of CNS sensory

TABLE 1 | Mutations of HCN1 and HCN2 in human epilepsy.

\begin{tabular}{lllll}
\hline Gene & Mutations & Phenotype & Biological effect & Reference \\
\hline HCN1 & G47V S100F S272P & $\begin{array}{l}\text { Early infantile epileptic } \\
\text { encephalopathy (Dravet-like } \\
\text { syndrome) }\end{array}$ & $\begin{array}{l}\text { Gain-of-function mutations with possible } \\
\text { dominant-negative effect (S100F, S272P, }\end{array}$ & Nava et al. (2014a) \\
H279Y R297T D401H & R297T) & Tang et al. (2008) \\
HCN2 & R527Q & Idiopathic generalized epilepsy & No significant variation of Ih current & Dibbens et al. (2010) \\
& delPPP & Febrile seizures (FS); genetic epilepsy & Gain-of-function mutation & DiFrancesco et al. (2011) \\
& with FS plus (GEFS+) & Loss-of-function mutation & Nakamura et al. (2013)
\end{tabular}



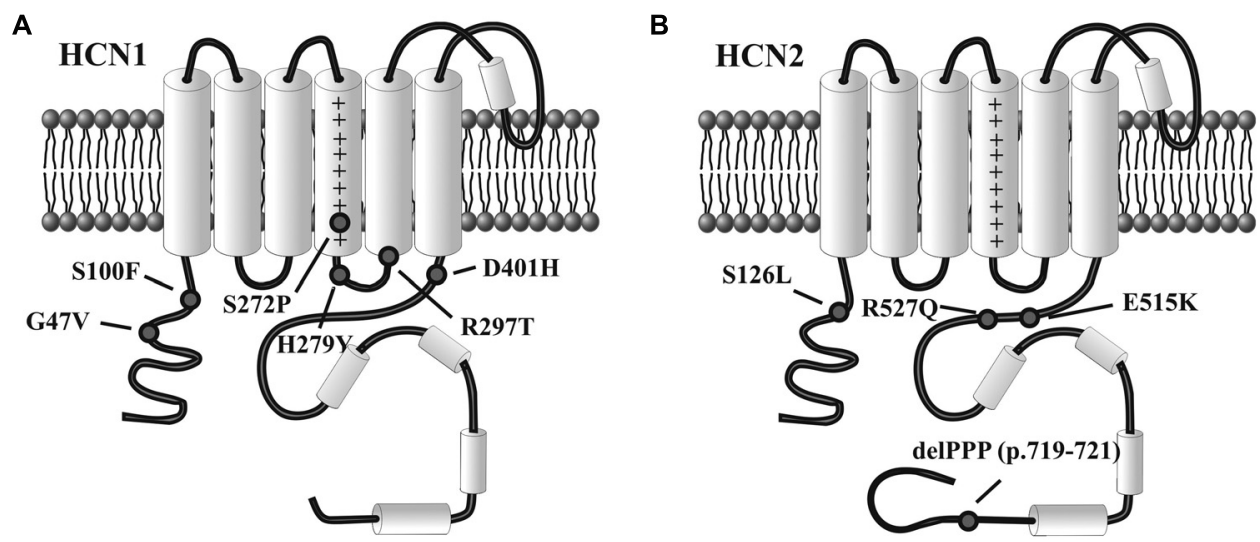

FIGURE 2 | Variants of HCN1 and HCN2 channels associated with epilepsy. Diagram representation of single human HCN1 (A) and HCN2 channel subunits (B) showing the approximate position of mutations linked to infantile epileptic encephalopathy (HCN1) or inheritable forms of epilepsy (HCN2), as reported in the literature. See text for further explanation.

processing is likely in neuropathic pain transmission, the condition of the injured peripheral nerve is critical for its initiation and maintenance.

There is increasing evidence demonstrating a crucial role played by the class of HCN ion channels in starting and controlling firing frequency of action potentials responsible for pain (Jiang et al., 2008). Although the HCN1 isoform is one of the pharmacological targets of the potent and widely used anesthetic propofol (Tibbs et al., 2013), its contribution to the transmission of pain seems to be limited. Recent experimental data have indeed demonstrated the strategic role played by HCN2 in both inflammatory and neuropathic pain, suggesting the selective blocking of its activity as a potential target for the treatment of pain.

\section{Inflammatory Pain}

It is established that the inhibition of the $\mathrm{I}_{\mathrm{h}}$ current, by drugs blocking HCN channels activity in a non-selective manner, prevents inflammatory pain generated in various animal models by different stimuli (Luo et al., 2007; Dunlop et al., 2009; Takasu et al., 2010). However, these results do not allow to explain the contribution of the specific channel isoforms involved in pain. Selective genetic deletion of different $\mathrm{HCN}$ isoforms recently clarified this important aspect.

According to studies in an HCN1 KO model, this channel isoform does not appear to play a crucial role in the transmission of inflammatory pain (Momin et al., 2008). HCN2 global KO models have not been tested for the studies of pain, since they are associated with severe neurological phenotypes, such as generalized epilepsy and ataxia, and are not prone to behavioral studies (Ludwig et al., 2003; Chung et al., 2009).

Recently, a genetic approach using the selective deletion of HCN2 in the Nav1.8 sensory neurons has contributed to clarify the important role played by HCN2 in the generation and maintenance of pain. The study showed that the perception of pain secondary to the administration of different inflammatory stimuli was lost in HCN2 KO mice, while the reaction to acute pain in the absence of inflammation remained unaltered. These data strongly suggest that block of $\mathrm{HCN} 2$ can be considered as a putatively very useful strategy for the treatment of inflammatory pain, without the adverse effect of reducing the perception of the normal acute stimulus (Emery et al., 2011, 2012).

\section{Neuropathic Pain}

Non-selective pharmacological block of HCN channels also inhibits neuropathic pain, suggesting their involvement in pain perception caused by direct nerve injury. The genetic deletion of $\mathrm{HCN} 1$ partially reduces the neuropathic pain determined by direct damage on the nerve (Momin et al., 2008), while the contribution of HCN2 appears to be more substantial. The animal model with a selective KO of HCN2 in Nav1.8-expressing peripheral neurons shows the absence of hyperalgesia following the application of different nociceptive stimuli that determine nerve injury. These results support the notion that the HCN2 isoform is a key factor in initiating neuronal excitability following nerve damage and in the maintenance of neuropathic pain. Accordingly, the selective inhibition of HCN2 activity could be expected to represent an interesting specific target for the treatment of this debilitating condition, widespread in the general population. However, given the key role played by HCN2 in the human CNS (DiFrancesco et al., 2011), any specific HCN2-blocking drug used as a therapy against neuropathic pain should be able to perfuse the peripheral nervous system, without passing across the blood brain barrier, in order to avoid serious neurological adverse consequence, such as seizures.

It is also worth noticing that ivabradine, the unique clinically approved non-selective HCN blocker used for coronary heart disease and heart failure (DiFrancesco and Camm, 2004; DiFrancesco and Borer, 2007) has been reported to reduce mechanical allodynia, inflammatory and neuropathic pain in animal models, further supporting the involvement of HCN channels in pain transmission (Noh et al., 2014; Young et al., 2014). However, until more isoform-specific drugs are commercially available, clinical use of ivabradine 
in the treatment of pain is obviously hindered by the bradycardic effect due to block of HCN4 activity in the heart.

\section{Parkinson's Disease}

Parkinson's disease (PD) is one of the most common neurodegenerative disease, characterized by progressive motor symptoms such as bradykinesia, rigidity, and resting tremor (Hammond et al., 2007; Ali and Morris, 2015). Progressive degeneration of dopamine (DA) neurons in the substantia nigra pars compacta $(\mathrm{SNc})$ is the main etiology of $\mathrm{PD}$.

HCN2 and HCN4 channels are highly expressed in midbrain DA neurons, where they participate in the regulation of network spontaneous activity (Mercuri et al., 1995; Santoro et al., 2000; Notomi and Shigemoto, 2004; Chu and Zhen, 2010).

Recent data acquired from genetic and pharmacological animal models of PD reported a progressive downregulation of HCN channel activity following DA depletion and DA neuronal loss, although channel protein expression did not appear to be affected (Good et al., 2011). Interestingly, the delivery of $\mathrm{HCN}$ subunits restored pacemaking activity and reduced burst spiking, even if the motor disability induced by DA depletion was not reversed (Chan et al., 2011). A role of HCN channel downregulation in PD is also supported by a computational model of the activity of the globus pallidus, which suggests a possible mechanism for the emergence of parkinsonian activity (Merrison-Hort and Borisyuk, 2013).

Recently, Masi et al. (2013) reported novel evidence for a possible influence of HCN channel dysfunction in the pathogenesis of human $\mathrm{PD}$. They showed that $\mathrm{MPP}^{+}$, a potent parkinsonizing agent well known as a blocker of the mitochondrial complex I, also leads to a dose-dependent inhibition of $\mathrm{I}_{\mathrm{h}}$ in SNc DA neurons. The electrophysiological analysis of this pharmacological model revealed a reduced spontaneous activity of $I_{h}$ in SNc DA neurons, characterized by an increased responsiveness toward synaptic excitation, possibly contributing to the pathogenesis of human PD.

\section{Conclusion}

HCN channels play a fundamental role in the control of neuronal excitability and network activity in the nervous system. Accumulating evidence supports the view that modifications in the HCN physiological function contribute to the pathogenic mechanism leading to certain neurological diseases in humans such as epilepsy, pain, and, as recently emerged, PD.

Although it is now clear that HCN channels play an important role in the pathogenesis of epilepsy, the precise mechanisms underlying their role in this common disease still remains to be elucidated. Thorough genetic studies of large populations of epileptic patients, able to associate the various forms of epilepsy (i.e., absence, IGE, TLE, various type of epileptic encephalopathies) to specific alterations of the channels, have yet to be performed. However, preliminary results today available suggest that $H C N 1$ point mutations leading to channel dysfunction are associated with severe and progressive epileptic diseases, while HCN2 mutations account for milder generalized phenotypes, without evidence of a progressive feature. Interestingly, there are no mutations of HCN channels reported in partial epilepsies TLE, although the experimental data from animal models allow to hypothesize their presence in humans. It will certainly be necessary to better understand the role played by dysfunctional HCN channels in predisposing to the development of the epileptogenic action potential. Understanding these aspects will contribute to identify novel potential therapeutic strategies for the treatment of epilepsy.

It is also important to note that the involvement of $\mathrm{HCN}$ channels in a wide variety of neuronal processes including sensory signal transduction, dendritic integration, synaptic plasticity, pacemaking, and network oscillations, motor learning, and others (Robinson and Siegelbaum, 2003) makes them likely contributors, when defective, to pathological behavior. As a natural consequence of their contribution as neurological disease-causing factors, HCN channels have long been considered an attractive therapeutic target (Postea and Biel, 2011; Reid et al., 2012; Shah et al., 2013). It is interesting to note in this context that, while most epilepsy-linked HCN mutations shown to generate hyperexcitability are loss-of-function (Lewis and Chetkovich, 2011), reduction of $\mathrm{I}_{\mathrm{h}}$ activity is known to reduce chronic pain (Emery et al., 2012; Young et al., 2014). The reason for this apparently paradoxical effect is that neuronal excitability depends both on input membrane resistance and resting membrane voltage, whose $\mathrm{I}_{\mathrm{h}}$-linked modifications affect excitability in opposite ways (Poolos et al., 2002). It will be crucial in the near future to clarify the details of $\mathrm{HCN}$-associated excitatory vs. inhibitory effects, in order to identify specific pharmacological strategies for these two clinical conditions, both extremely common in the general population and poorly treated by conventional drug therapy, and often characterized by multiple side effects.

The understanding of the processes rendering HCN channels dysfunctional in human neurological diseases will provide in the future further important insight into the molecular mechanisms responsible for the disease, a concept likely to be applicable to a larger number of ion channelopathies than is known to date. While studies of animal models can clearly provide essential basic information, it is highly desirable that further investigation is focused on the identification of the role played by HCN channelopathies in human diseases. These studies should be eventually aimed at the development of selective pharmacological strategies specifically targeting $\mathrm{HCN}$ channels for the treatment of the neurological diseases.

\section{Acknowledgment}

This work was supported by the Italian Ministry of Health (Ricerca Finalizzata Giovani Ricercatori 2010), Project GR-20102304834 to JD. 


\section{References}

Ali, K., and Morris, H. R. (2015). Parkinson's disease: chameleons and mimics. Pract. Neurol. 15, 14-25. doi: 10.1136/practneurol-2014-000849

Avanzini, G., Franceschetti, S., and Mantegazza, M. (2007). Epileptogenic channelopathies: experimental models of human pathologies. Epilepsia 48(Suppl. 2), 51-64. doi: 10.1111/j.1528-1167.2007.01067.x

Barbuti, A., Baruscotti, M., and DiFrancesco, D. (2007a). The pacemaker current: from basics to the clinics. J. Cardiovasc. Electrophysiol. 18, 342-347. doi: 10.1111/j.1540-8167.2006.00736.x

Barbuti, A., Terragni, B., Brioschi, C., and DiFrancesco, D. (2007b). Localization of f-channels to caveolae mediates specific beta2-adrenergic receptor modulation of rate in sinoatrial myocytes. J. Mol. Cell. Cardiol. 42, 71-78. doi: 10.1016/j.yjmcc.2006.09.018

Baruscotti, M., Bottelli, G., Milanesi, R., DiFrancesco, J. C., and DiFrancesco, D. (2010). HCN-related channelopathies. Pflugers Arch. 460, 405-415. doi: 10.1007/s00424-010-0810-8

Bender, R. A., Soleymani, S. V., Brewster, A. L., Nguyen, S. T., Beck, H., Mathern, G. W., et al. (2003). Enhanced expression of a specific hyperpolarizationactivated cyclic nucleotide-gated cation channel (HCN) in surviving dentate gyrus granule cells of human and experimental epileptic hippocampus. J. Neurosci. 23, 6826-6836

Biel, M., Wahl-Schott, C., Michalakis, S., and Zong, X. (2009). Hyperpolarizationactivated cation channels: from genes to function. Physiol. Rev. 89, 847-885. doi 10.1152/physrev.00029.2008

Brewster, A., Bender, R. A., Chen, Y., Dube, C., Eghbal-Ahmadi, M., and Baram, T. Z. (2002). Developmental febrile seizures modulate hippocampal gene expression of hyperpolarization-activated channels in an isoform- and cellspecific manner. J. Neurosci. 22, 4591-4599.

Chan, C. S., Glajch, K. E., Gertler, T. S., Guzman, J. N., Mercer, J. N., Lewis, A. S., et al. (2011). HCN channelopathy in external globus pallidus neurons in models of Parkinson's disease. Nat. Neurosci. 14, 85-92. doi: 10.1038/nn.2692

Chu, H. Y., and Zhen, X. (2010). Hyperpolarization-activated, cyclic nucleotidegated $(\mathrm{HCN})$ channels in the regulation of midbrain dopamine systems. Acto Pharmacol. Sin. 31, 1036-1043. doi: 10.1038/aps.2010.105

Chung, W. K., Shin, M., Jaramillo, T. C., Leibel, R. L., Leduc, C. A., Fischer S. G., et al. (2009). Absence epilepsy in apathetic, a spontaneous mutant mouse lacking the h channel subunit, HCN2. Neurobiol. Dis. 33, 499-508. doi: 10.1016/j.nbd.2008.12.004

Depienne, C., Bouteiller, D., Keren, B., Cheuret, E., Poirier, K., Trouillard, O., et al. (2009a). Sporadic infantile epileptic encephalopathy caused by mutations in PCDH19 resembles Dravet syndrome but mainly affects females. PLoS Genet. 5:e1000381. doi: 10.1371/journal.pgen.1000381

Depienne, C., Trouillard, O., Saint-Martin, C., Gourfinkel-an, I., Bouteiller, D., Carpentier, W., et al. (2009b). Spectrum of SCN1A gene mutations associated with Dravet syndrome: analysis of 333 patients. J. Med. Genet. 46, 183-191. doi 10.1136/jmg.2008.062323

Dibbens, L. M., Reid, C. A., Hodgson, B., Thomas, E. A., Phillips, A. M., Gazina, E., et al. (2010). Augmented currents of an HCN2 variant in patients with febrile seizure syndromes. Ann. Neurol. 67, 542-546. doi: 10.1002/ana.21909

DiFrancesco, D. (1993). Pacemaker mechanisms in cardiac tissue. Annu. Rev. Physiol. 55, 455-472. doi: 10.1146/annurev.ph.55.030193.002323

DiFrancesco, D. (1999). Dual allosteric modulation of pacemaker (f) channels by cAMP and voltage in rabbit SA node. J. Physiol. 515, 367-376. doi: 10.1111/j.1469-7793.1999.367ac.x

DiFrancesco, D. (2006). Serious workings of the funny current. Prog. Biophys. Mol. Biol. 90, 13-25. doi: 10.1016/j.pbiomolbio.2005.05.001

DiFrancesco, D., and Borer, J. S. (2007). The funny current: cellular basis for the control of heart rate. Drugs 67(Suppl. 2), 15-24. doi: 10.2165/00003495200767002-00003

DiFrancesco, D., and Camm, J. A. (2004). Heart rate lowering by specific and selective I(f) current inhibition with ivabradine: a new therapeutic perspective in cardiovascular disease. Drugs 64, 1757-1765. doi: 10.2165/00003495200464160-00003

DiFrancesco, J. C., Barbuti, A., Milanesi, R., Coco, S., Bucchi, A., Bottelli, G., et al. (2011). Recessive loss-of-function mutation in the pacemaker HCN2 channel causing increased neuronal excitability in a patient with idiopathic generalized epilepsy. J. Neurosci. 31, 17327-17337. doi: 10.1523/JNEUROSCI.3727-11.2011
Dube, C., Chen, K., Eghbal-Ahmadi, M., Brunson, K., Soltesz, I., and Baram, T. Z. (2000). Prolonged febrile seizures in the immature rat model enhance hippocampal excitability long term. Ann. Neurol. 47, 336-344. doi: 10.1002/15318249(200003)47:3<336::AID-ANA9>3.0.CO;2-W

Dunlop, J., Vasilyev, D., Lu, P., Cummons, T., and Bowlby, M. R. (2009). Hyperpolarization-activated cyclic nucleotide-gated (HCN) channels and pain. Curr. Pharm. Des. 15, 1767-1772. doi: 10.2174/138161209788 186281

Dyhrfjeld-Johnsen, J., Morgan, R. J., and Soltesz, I. (2009). Double trouble? Potential for hyperexcitability following both channelopathic upand downregulation of $\mathrm{I}(\mathrm{h})$ in epilepsy. Front. Neurosci. 3:25-33. doi: 10.3389/neuro.01.005.2009

Emery, E. C., Young, G. T., Berrocoso, E. M., Chen, L., and Mcnaughton, P. A. (2011). HCN2 ion channels play a central role in inflammatory and neuropathic pain. Science 333, 1462-1466. doi: 10.1126/science.1206243

Emery, E. C., Young, G. T., and Mcnaughton, P. A. (2012). HCN2 ion channels: an emerging role as the pacemakers of pain. Trends Pharmacol. Sci. 33, 456-463. doi: 10.1016/j.tips.2012.04.004

Good, C. H., Hoffman, A. F., Hoffer, B. J., Chefer, V. I., Shippenberg, T. S., Bäckman, C. M., et al. (2011). Impaired nigrostriatal function precedes behavioral deficits in a genetic mitochondrial model of Parkinson's disease. FASEB J. 25, 1333-1344. doi: 10.1096/fj.10-173625

Gravante, B., Barbuti, A., Milanesi, R., Zappi, I., Viscomi, C., and DiFrancesco, D. (2004). Interaction of the pacemaker channel HCN1 with filamin A. J. Biol. Chem. 279, 43847-43853. doi: 10.1074/jbc.M401598200

Hammond, C., Bergman, H., and Brown, P. (2007). Pathological synchronization in Parkinson's disease: networks, models and treatments. Trends Neurosci. 30, 357-364. doi: 10.1016/j.tins.2007.05.004

Huang, Z., Walker, M. C., and Shah, M. M. (2009). Loss of dendritic HCN1 subunits enhances cortical excitability and epileptogenesis. J. Neurosci. 29, 10979-10988. doi: 10.1523/JNEUROSCI.1531-09.2009

Jiang, Y. Q., Sun, Q., Tu, H. Y., and Wan, Y. (2008). Characteristics of HCN channels and their participation in neuropathic pain. Neurochem. Res. 33, 1979-1989. doi: 10.1007/s11064-008-9717-6

Jung, S., Jones, T. D., Lugo, J. N., Sheerin, A. H., Miller, J. W., D’ambrosio, R., et al. (2007). Progressive dendritic HCN channelopathy during epileptogenesis in the rat pilocarpine model of epilepsy. J. Neurosci. 27, 13012-13021. doi: 10.1523/JNEUROSCI.3605-07.2007

Jung, S., Warner, L. N., Pitsch, J., Becker, A. J., and Poolos, N. P. (2011). Rapid loss of dendritic HCN channel expression in hippocampal pyramidal neurons following status epilepticus. J. Neurosci. 31, 14291-14295. doi 10.1523/JNEUROSCI.1148-11.2011

Kimura, K., Kitano, J., Nakajima, Y., and Nakanishi, S. (2004). Hyperpolarizationactivated, cyclic nucleotide-gated $\mathrm{HCN} 2$ cation channel forms a protein assembly with multiple neuronal scaffold proteins in distinct modes of protein-protein interaction. Genes Cells 9, 631-640. doi: 10.1111/j.13569597.2004.00752.x

Kole, M. H., Bräuer, A. U., and Stuart, G. J. (2007). Inherited cortical HCN1 channel loss amplifies dendritic calcium electrogenesis and burst firing in a rat absence epilepsy model. J. Physiol. 578, 507-525. doi: 10.1113/jphysiol.2006.122028

Lewis, A. S., and Chetkovich, D. M. (2011). HCN channels in behavior and neurological disease: too hyper or not active enough? Mol. Cell. Neurosci. 46, 357-367. doi: 10.1016/j.mcn.2010.11.007

Linley, J. E., Rose, K., Ooi, L., and Gamper, N. (2010). Understanding inflammatory pain: ion channels contributing to acute and chronic nociception. Pflugers Arch. 459, 657-669. doi: 10.1007/s00424-010-0784-6

Ludwig, A., Budde, T., Stieber, J., Moosmang, S., Wahl, C., Holthoff, K., et al. (2003). Absence epilepsy and sinus dysrhythmia in mice lacking the pacemaker channel HCN2. EMBO J. 22, 216-224. doi: 10.1093/emboj/cdg032

Luo, L., Chang, L., Brown, S. M., Ao, H., Lee, D. H., Higuera, E. S., et al. (2007). Role of peripheral hyperpolarization-activated cyclic nucleotide-modulated channel pacemaker channels in acute and chronic pain models in the rat. Neuroscience 144, 1477-1485. doi: 10.1016/j.neuroscience.2006.10.048

Mantegazza, M., Rusconi, R., Scalmani, P., Avanzini, G., and Franceschetti, S. (2010). Epileptogenic ion channel mutations: from bedside to bench and, hopefully, back again. Epilepsy Res. 92, 1-29. doi: 10.1016/j.eplepsyres.2010.08.003

Masi, A., Narducci, R., Landucci, E., Moroni, F., and Mannaioni, G. (2013). $\mathrm{MPP}(+)$-dependent inhibition of Ih reduces spontaneous activity and 
enhances EPSP summation in nigral dopamine neurons. Br. J. Pharmacol. 169, 130-142. doi: 10.1111/bph.12104

Mercuri, N. B., Bonci, A., Calabresi, P., Stefani, A., and Bernardi, G. (1995) Properties of the hyperpolarization-activated cation current Ih in rat midbrain dopaminergic neurons. Eur. J. Neurosci. 7, 462-469. doi: 10.1111/j.14609568.1995.tb00342.x

Merrison-Hort, R., and Borisyuk, R. (2013). The emergence of two anti-phase oscillatory neural populations in a computational model of the Parkinsonian globus pallidus. Front. Comput. Neurosci. 7:173. doi: 10.3389/fncom.2013. 00173

Michels, G., Er, F., Khan, I. F., Endres-Becker, J., Brandt, M. C., Gassanov, N., et al. (2008). K+ channel regulator KCR1 suppresses heart rhythm by modulating the pacemaker current If. PLOS ONE 3:e1511. doi: 10.1371/journal.pone. 0001511

Momin, A., Cadiou, H., Mason, A., and Mcnaughton, P. A. (2008). Role of the hyperpolarization-activated current Ih in somatosensory neurons. J. Physiol. 586, 5911-5929. doi: 10.1113/jphysiol.2008.163154

Munsch, T., and Pape, H. C. (1999). Upregulation of the hyperpolarizationactivated cation current in rat thalamic relay neurones by acetazolamide. J. Physiol. 519, 505-514. doi: 10.1111/j.1469-7793.1999. 0505m.x

Nakamura, Y., Shi, X., Numata, T., Mori, Y., Inoue, R., Lossin, C., et al. (2013). Novel HCN2 mutation contributes to febrile seizures by shifting the channel's kinetics in a temperature-dependent manner. PLoS ONE 8:e80376. doi: 10.1371/journal.pone.0080376

Nava, C., Dalle, C., Rastetter, A., Striano, P., De Kovel, C. G., Nabbout, R., et al. (2014a). De novo mutations in HCN1 cause early infantile epileptic encephalopathy. Nat. Genet. 46, 640-645. doi: 10.1038/ng.2952

Nava, C., Keren, B., Mignot, C., Rastetter, A., Chantot-Bastaraud, S., Faudet, A., et al. (2014b). Prospective diagnostic analysis of copy number variants using SNP microarrays in individuals with autism spectrum disorders. Eur. J. Hum. Genet. 22, 71-78. doi: 10.1038/ejhg.2013.88

Nawathe, P. A., Kryukova, Y., Oren, R. V., Milanesi, R., Clancy, C. E., Lu, J. T., et al. (2013). An LQTS6 MiRP1 mutation suppresses pacemaker current and is associated with sinus bradycardia. J. Cardiovasc. Electrophysiol. 24, 1021-1027. doi: $10.1111 /$ jce. 12163

Noam, Y., Ehrengruber, M. U., Koh, A., Feyen, P., Manders, E. M., Abbott, G. W., et al. (2014). Filamin A promotes dynamin-dependent internalization of hyperpolarization-activated cyclic nucleotide-gated type 1 (HCN1) channels and restricts Ih in hippocampal neurons. J. Biol. Chem. 289, 5889-5903. doi: 10.1074/jbc.M113.522060

Noh, S., Kumar, N., Bukhanova, N., Chen, Y., Stemkowsi, P. L., and Smith, P. A. (2014). The heart-rate-reducing agent, ivabradine, reduces mechanical allodynia in a rodent model of neuropathic pain. Eur. J. Pain 18, 1139-1147. doi: 10.1002/j.1532-2149.2014.00460.x

Nolan, M. F., Malleret, G., Dudman, J. T., Buhl, D. L., Santoro, B., Gibbs, E., et al. (2004). A behavioral role for dendritic integration: HCN1 channels constrain spatial memory and plasticity at inputs to distal dendrites of CA1 pyramidal neurons. Cell 119, 719-732.

Notomi, T., and Shigemoto, R. (2004). Immunohistochemical localization of Ih channel subunits, HCN1-4, in the rat brain. J. Comp. Neurol. 471, 241-276. doi: 10.1002/cne.11039

Pape, H. C. (1996). Queer current and pacemaker: the hyperpolarizationactivated cation current in neurons. Annu. Rev. Physiol. 58, 299-327. doi: 10.1146/annurev.ph.58.030196.001503

Phillips, A. M., Kim, T., Vargas, E., Petrou, S., and Reid, C. A. (2014). Spike-andwave discharge mediated reduction in hippocampal HCN1 channel function associates with learning deficits in a genetic mouse model of epilepsy. Neurobiol. Dis. 64, 30-35. doi: 10.1016/j.nbd.2013.12.007

Poolos, N. P., Migliore, M., and Johnston, D. (2002). Pharmacological upregulation of h-channels reduces the excitability of pyramidal neuron dendrites. Nat. Neurosci. 5, 767-774. doi: 10.1038/nn891

Postea, O., and Biel, M. (2011). Exploring HCN channels as novel drug targets. Nat. Rev. Drug Discov. 10, 903-914. doi: 10.1038/nrd3576

Powell, K. L., Ng, C., O’brien, T. J., Xu, S. H., Williams, D. A., Foote, S. J., et al. (2008). Decreases in HCN mRNA expression in the hippocampus after kindling and status epilepticus in adult rats. Epilepsia 49, 1686-1695. doi: 10.1111/j.1528-1167.2008.01593.x
Reid, C. A., Phillips, A. M., and Petrou, S. (2012). HCN channelopathies: pathophysiology in genetic epilepsy and therapeutic implications. Br. J. Pharmacol. 165, 49-56. doi: 10.1111/j.1476-5381.2011.01507.x

Richichi, C., Brewster, A. L., Bender, R. A., Simeone, T. A., Zha, Q., Yin, H. Z., et al. (2008). Mechanisms of seizure-induced 'transcriptional channelopathy' of hyperpolarization-activated cyclic nucleotide gated (HCN) channels. Neurobiol. Dis. 29, 297-305. doi: 10.1016/j.nbd.2007. 09.003

Robinson, R. B., and Siegelbaum, S. A. (2003). Hyperpolarization-activated cation currents: from molecules to physiological function. Annu. Rev. Physiol. 65, 453-480. doi: 10.1146/annurev.physiol.65.092101.142734

Santoro, B., Chen, S., Luthi, A., Pavlidis, P., Shumyatsky, G. P., Tibbs, G. R., et al. (2000). Molecular and functional heterogeneity of hyperpolarizationactivated pacemaker channels in the mouse CNS. J. Neurosci. 20, 5264-5275.

Santoro, B., Lee, J. Y., Englot, D. J., Gildersleeve, S., Piskorowski, R. A., Siegelbaum, S. A., et al. (2010). Increased seizure severity and seizure-related death in mice lacking HCN1 channels. Epilepsia 51, 1624-1627. doi: 10.1111/j.15281167.2010.02554.x

Santoro, B., Liu, D. T., Yao, H., Bartsch, D., Kandel, E. R., Siegelbaum, S. A., et al. (1998). Identification of a gene encoding a hyperpolarization-activated pacemaker channel of brain. Cell 93, 717-729. doi: 10.1016/S0092-8674(00) 81434-8

Santoro, B., Piskorowski, R. A., Pian, P., Hu, L., Liu, H., and Siegelbaum, S. A. (2009). TRIP8b splice variants form a family of auxiliary subunits that regulate gating and trafficking of HCN channels in the brain. Neuron 62, 802-813. doi: 10.1016/j.neuron.2009.05.009

Santoro, B., Wainger, B. J., and Siegelbaum, S. A. (2004). Regulation of HCN channel surface expression by a novel C-terminal protein-protein interaction. J. Neurosci. 24, 10750-10762. doi: 10.1523/JNEUROSCI.3300-04.2004

Shah, M. M., Huang, Z., and Martinello, K. (2013). HCN and KV7 (M-) channels as targets for epilepsy treatment. Neuropharmacology 69, 75-81. doi: 10.1016/j.neuropharm.2012.03.005

Surges, R., Freiman, T. M., and Feuerstein, T. J. (2003). Gabapentin increases the hyperpolarization-activated cation current Ih in rat CA1 pyramidal cells. Epilepsia 44, 150-156. doi: 10.1046/j.1528-1157.2003.36802.x

Takasu, K., Ono, H., and Tanabe, M. (2010). Spinal hyperpolarization-activated cyclic nucleotide-gated cation channels at primary afferent terminals contribute to chronic pain. Pain 151, 87-96. doi: 10.1016/j.pain.2010.06.020

Tang, B., Sander, T., Craven, K. B., Hempelmann, A., and Escayg, A. (2008). Mutation analysis of the hyperpolarization-activated cyclic nucleotide-gated channels HCN1 and HCN2 in idiopathic generalized epilepsy. Neurobiol. Dis. 29, 59-70. doi: 10.1016/j.nbd.2007.08.006

Thomas, R. H., and Berkovic, S. F. (2014). The hidden genetics of epilepsya clinically important new paradigm. Nat. Rev. Neurol. 10, 283-292. doi: 10.1038/nrneurol.2014.62

Tibbs, G. R., Rowley, T. J., Sanford, R. L., Herold, K. F., Proekt, A., Hemmings, H. C., et al. (2013). HCN1 channels as targets for anesthetic and nonanesthetic propofol analogs in the amelioration of mechanical and thermal hyperalgesia in a mouse model of neuropathic pain. J. Pharmacol. Exp. Ther. 345, 363-373. doi: 10.1124/jpet.113.203620

Tsay, D., Dudman, J. T., and Siegelbaum, S. A. (2007). HCN1 channels constrain synaptically evoked $\mathrm{Ca}^{2+}$ spikes in distal dendrites of CA1 pyramidal neurons. Neuron 56, 1076-1089. doi: 10.1016/j.neuron.2007.11.015

Wickenden, A. D., Maher, M. P., and Chaplan, S. R. (2009). HCN pacemaker channels and pain: a drug discovery perspective. Curr. Pharm. Des. 15, 2149-2168. doi: 10.2174/138161209788489122

Ying, S. W., Kanda, V. A., Hu, Z., Purtell, K., King, E. C., Abbott, G. W., et al. (2012). Targeted deletion of Kcne2 impairs HCN channel function in mouse thalamocortical circuits. PLoS ONE 7:e42756. doi: 10.1371/journal.pone.0042756

Young, G. T., Emery, E. C., Mooney, E. R., Tsantoulas, C., and Mcnaughton, P. A. (2014). Inflammatory and neuropathic pain are rapidly suppressed by peripheral block of hyperpolarisation-activated cyclic nucleotide-gated ion channels. Pain 155, 1708-1719. doi: 10.1016/j.pain.2014.05.021

Yu, H., Wu, J., Potapova, I., Wymore, R. T., Holmes, B., Zuckerman, J., et al. (2001). MinK-related peptide 1: a beta subunit for the HCN ion channel subunit family enhances expression and speeds activation. Circ. Res. 88, E84-E87. doi: $10.1161 /$ hh1201.093511 
Zagotta, W. N., Olivier, N. B., Black, K. D., Young, E. C., Olson, R., and Gouaux, E. (2003). Structural basis for modulation and agonist specificity of HCN pacemaker channels. Nature 425, 200-205. doi: 10.1038/nature01922

Conflict of Interest Statement: The authors declare that the research was conducted in the absence of any commercial or financial relationships that could be construed as a potential conflict of interest.
Copyright (c) 2015 DiFrancesco and DiFrancesco. This is an open-access article distributed under the terms of the Creative Commons Attribution License (CC BY). The use, distribution or reproduction in other forums is permitted, provided the original author(s) or licensor are credited and that the original publication in this journal is cited, in accordance with accepted academic practice. No use, distribution or reproduction is permitted which does not comply with these terms. 